\title{
Web-Based Interactive Tool to Support Shared Decision-Making in the Choice of Treatment for Advanced Pancreatic Cancer
}

\author{
Ejike T. Ezeh \\ Bournemouth University \\ Bournemouth, United Kingdom \\ eezeh@bournemouth.ac.uk
}

\begin{abstract}
Pancreatic cancer currently has no cure in its advanced stage and often, systemic chemotherapy is used to improve survival and quality of life of patients. Chemotherapy treatments are available with varying degrees of benefits, harms, and uncertainties, and patients need to be aware of these characteristics and make informed choices or decide whether to avoid chemotherapy altogether. Web-based tools may help patients in making this informed choice with the help of their clinicians and relatives. Therefore, the aim of the study is to investigate the potential of a web-based interactive tool to support shared decision-making in the choice of treatment for people with advanced pancreatic cancer. A four-phase mixed methods paradigm has been adopted for the study. Participants will be clinicians (oncologists, clinical nurse specialists), people diagnosed with advanced (inoperable) pancreatic cancer, and relatives who support these patients. The first phase of the study identified 60 relevant citations on advanced pancreatic cancer treatment from four electronic databases from 1997 to 2018.
\end{abstract}

Pancreatic cancer, decision aid, mixed methods research, web-based interactive tool, shared decisionmaking, human-centred design

\section{INTRODUCTION}

Pancreatic cancer $(\mathrm{PC})$ is a malignant tumour that accounts for a projected mortality of over 87,000 in the European Union (EU) in 2017 (Malvezzi et al. 2017). It is currently the fourth leading cause of death from cancer in the EU (Ferlay et al. 2016). In England, 5-year survival rate is less than 10\% (Office for National Statistics 2017). PC be generally classified as resectable, borderline resectable, locally advanced, and metastatic (Vincent et al. 2011). Treatments include surgery, chemotherapy, radiation therapy, and palliative care (Kamisawa et al. 2016). Surgery offers curative treatment, but $80 \%$ of patients are diagnosed in the advanced stage (that is, locally advanced or metastatic) and are ineligible for curative surgery (Ducreux et al. 2015, Taieb et al. 2017). However, systemic chemotherapy is a palliative option for people with advanced pancreatic cancer (APC) (Balaban et al. 2016). "Best supportive care" (BSC), or "supportive care", is another option which involves symptom management and improving quality of life (Hui et al. 2013).
Several chemotherapy treatments are available for APC (Tempero et al. 2017), and it is helpful for patients and their relatives to be adequately informed of the benefits and harms associated with these treatments. They have the right to make the decision about whether to embark on a chemotherapy regime or to opt for BSC based on their individual preferences. It is also desirable for clinicians to have a means with which to communicate with patients about their illness and how to elicit their preferences on what matters most to them during this difficult period of prognosis. One way to provide this convergence between clinicians and patients is through shared decision-making (SDM), which is "an approach where clinicians and patients make decisions together using the best available evidence" (Elwyn et al. 2010, Stiggelbout et al. 2012).

In July 2016, a prototype web-based tool on advanced cancer treatment options was tested by researchers at Bournemouth University among a small group of oncologists, lead cancer nurses, and service users. It received positive feedback with suggestions for the current research. 
Therefore, the purpose of the study is to investigate the potential of a web-based interactive tool in SDM between people affected with APC and their clinicians, based on available clinical evidence and preferences of these people.

\section{RESEARCH OBJECTIVES}

(i) To assess the comparative efficacy, toxicity, and quality-of-life characteristics of chemotherapy treatments for APC as reported in randomized controlled trials (RCTs) through a systematic review and network meta-analysis of these RCTs.

(ii) To explore the expectations and preferences of clinicians, people with APC, and their relatives, when making decisions about treatment, through focus groups and semi-structured interviews for clinicians, and people with APC (including their relatives), respectively.

(iii) To identify features necessary for developing a web-based interactive tool to facilitate SDM between clinicians and people with APC about choice of treatment.

(iv) To evaluate the effectiveness of the webbased interactive tool in SDM, through a pilot test with clinicians (doctors and specialist nurses), people with APC, and their relatives.

\section{KEY POINTS OF THE LITERATURE REVIEW}

\subsection{Shared decision-making in treatment deliberation}

Decision making is an important component of the medical encounter. Different decision models exist: paternalistic, informed, and shared models of decision-making (Charles et al. 1997, Charles et al. 1999). However, the shared model of decisionmaking, or SDM, has gained prominence in recent times because it supports the person-centred philosophy in healthcare(Coulter and Collins 2011).

SDM is encouraged in the United Kingdom (UK), United States (US) and Canada(Elwyn et al. 2010).

It is rooted in the principles of biomedical ethics (Beauchamp and Childress 2013). These principles guide how the medical practice relates with patients in healthcare provision. Most patients would want to be more involved in decisions about the treatment they receive(Stacey et al. 2008).

Clinical equipoise is the existence of more than one treatment for a health condition having varying degrees of benefit and harm (Freedman 1987). The concept of clinical equipoise makes SDM possible in medical deliberations (Elwyn et al. 2000, Elwyn et al. 2009). Balaban et al. (2016) alluded to the presence of clinical equipoise in APC treatment. Evidence shows that doctors are not the best judges of patients' preferences (Hofmann et al. 1997), therefore, there is need for a partnership of more than one proficiency (doctors with medical expertise, and patients with experiential expertise).

\subsection{Patient decision aids in the medical encounter}

Patient decision aids (PtDAs) are standardized, evidence-based tools used to facilitate SDM (O'Connor et al. 2004). They have the following general functions: explicit statement of the decision to be made, evidence-based information about the condition of interest, and ability to help users clarify their values in reference to the benefits and harms inherent in the available options of treatment (Stacey et al. 2017).

PtDAs have been developed for quite a few health conditions, either for screening decisions or treatment choice (Stacey et al. 2017).

PtDAs can be used before, during, or after the consultation process (Stacey et al. 2017). They could be in the form of pamphlets, video, or they may be internet-based (O'Connor et al. 2004, Coulter and Collins 2011, Stiggelbout et al. 2012).

Reviews have shown that PtDAs are effective in improving patients' knowledge about their condition, and participation in the medical encounter (Stacey et al. 2017).

\subsection{Web-based patient decision aids}

There has been substantial growth in web-based PtDAs in recent times; from four randomized studies identified in 2011 (Stacey et al. 2011) to more than twenty in 2017 (Stacey et al. 2017).

Some obvious benefits of web-based PtDAs are: easy access via a browser, personalized information display control, and they can be developed to collect and manage user preferences on-the-go.

According to Hoffman et al. (2013), several theories underpin the delivery of patient decision aids on the internet. These include theories from cognitive psychology, decision psychology, and communication.

Various development frameworks were compared and a new model was proposed for the systematic development of decision aids by Coulter et al.Coulter et al. (2013). Elwyn et al. developed a process map for development of decision support interventions for patients (2011). However, these processes are quite involved and complex.

Eleven main issues were identified that should be addressed when presenting data in decision aids 
(Trevena et al. 2013). However, there are no generally agreed methods of developing internetbased decision tools in healthcare.

A recent systematic review identified and analysed 58 studies involving computer based decision aids (Syrowatka et al. 2016). The following features were common among the decision aids: content control, tailoring, patient narratives, explicit values clarification, feedback, and social support. These features had contrasting impact on the quality of decision-making.

A web-based tool for informed decision-making was developed for people with APC in Canada (Gresham 2013). However, this tool provided limited options to users and it was designed for patients in the context of informed decision. To the best of our knowledge, no web-based tool exists to facilitate SDM for treatment in APC, for patients, their clinicians and relatives.

This research aims to add to the existing knowledge and leverage the benefits offered by technology in the design and development of a web-based interactive tool to facilitate SDM in choice of treatment for APC. It is hoped that knowledge acquired here will be transferable to the development of similar tools for other cancer types.

\section{PROBLEM STATEMENT}

\subsection{Current state}

Deliberations are usually accompanied with (or without) paper decision aids. Several websites are also available with general information for patients. However, information provided is too broad, there are no interactive tools to assess and clarify values, and there are no means of information management by the users or involving their relatives in the decision-making process.

\subsection{Ideal state}

The potential for a more engaging and informative consultation for all parties during the consultation process, thereby facilitating SDM, leading to more effective clinical encounters.

\subsection{Proposed solution}

A web-based interactive tool to support treatment choice of people with APC. This is beyond a website with information on pancreatic cancer. It will include preference-clarifying components to assist users in their decision-making, and invitation of other members of one's family to help with difficult decisions and clarifications. A recent randomized control trial showed that a web-based decision aid performed better than a website in preparing patients for decision about clinical trial participation (Politi et al. 2016).

\section{RESEARCH APPROACH AND METHODOLOGY}

The research involves the development of a webbased interactive system with multiuser capabilities. It also requires inputs from various disciplines (health science, information technology, and sociology) to meet its design goals.

The mixed methods research paradigm, which will be the overarching design framework, is a research methodology that combines both quantitative and qualitative data in answering research questions. Some of the reasons for choosing this methodology are: the need to explore before administering instruments, the need to involve participants in the study, the need to develop, implement and evaluate (Creswell and Plano Clark 2017).

Adaptations to the models proposed by Coulter et al. (2013) and Elwyn et al. (2011) will guide the research design. Certain basic similarities can be observed when these two models are compared, namely: scoping or content specification, evidence synthesis, tool development, and testing.

Based on the preceding, four major phases were identified for the implementation of the research objectives. They are (1) evidence synthesis, (2) end-user needs assessment, (3) tool development, and (4) end-user evaluation.

The research design is illustrated in Figure 1.

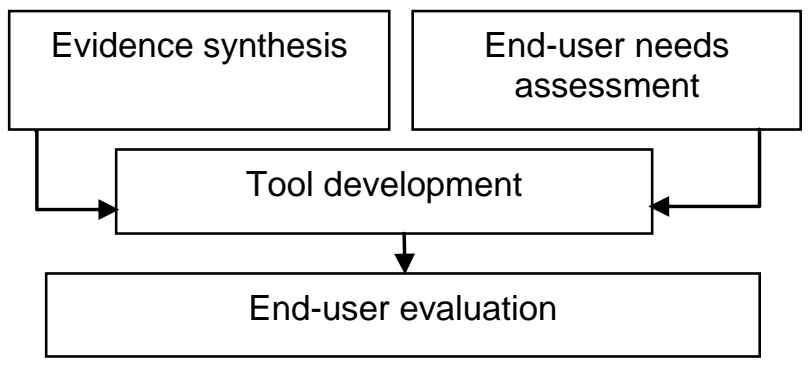

Figure 1: Diagram of Research Design

\subsection{Phase 1: Evidence Synthesis}

This phase involves a systematic review and network meta-analysis (NMA) of phase 3 randomized controlled trials on APC treatment with chemotherapy as a first-line option. NMA or multiple treatment comparison involves the statistical combination of treatment effects from different studies that have not directly compared treatments of interest in a randomized controlled trial (Lumley 2002, Lu and Ades 2004). This affords statistical possibility in combining similar trials in a 
single network and making comparisons on different outcomes of interest.

The Preferred Reporting Items for Systematic Reviews and Meta-analysis (PRISMA) (Moher et al. 2009)will be used a guideline for the reporting the results of this phase.

The outcome of this phase will be a synthesis of information from studies on efficacy, toxicity and quality of life for different chemotherapy regimens. The data from this phase will provide evidence to inform decision-making for users of the interactive tool.

\subsubsection{Eligibility of included studies}

Studies to be included in the systematic review will be phase 3 RCTs of advanced pancreatic cancer (locally advanced or metastatic) chemotherapy treatment from 1997 to 2018.

Inclusion criteria: first-line chemotherapy treatment for APC

Exclusion criteria: single-arm studies, studies comparing radiotherapy, adjuvant chemotherapy studies.

\subsection{Phase 2: End-user needs assessment}

This phase will explore the experiences and expectations of clinicians, patients, and their relatives about APC treatment and how a webbased tool could help them in this process. Interviews and focus groups doctors, specialist nurses, people with APC and their relatives. There is no agreement in the procedure for carrying out needs assessment for participants in the development of a decision aid (Coulter et al. 2013). However, the use of focus groups and interviews are widely employed for eliciting user needs in similar studies (Leighl et al. 2008, Ozanne et al. 2015).

Thematic content analysis of the data collected will potentially yield some themes that will form the basis for some of the requirements specification for the proposed interactive tool.

\subsection{Phase 3: Tool development}

Information gathered from phase 1 and 2 will be used to design and develop a web-based, interactive tool to facilitate SDM between clinicians and people with APC.

In addition to the outcome of the themes synthesized from phase 2, some of the potential functionalities of the proposed tool will be:

(i) Statistical ranking of treatment options in terms of efficacy, quality of life, or toxicity. (ii) Elicitation and clarification of user preference to help inform choice of the most appropriate treatment.

(iii) Clinician information entry based on what they perceive of the patient's current condition and comparison of clinicianpatient preferences.

(iv) Collaboration between patients and their relatives about their preferences and suggestions to help the patients in decision-making.

(v) Assessment of user knowledge in relation to their choice of treatment through values clarification exercise.

These are based on a cursory observation of similar tools.

Human-centred design (Standardization IOF 2010, Giacomin 2014) approach will guide the development of the interactive tool. This approach places more focus on humans as primary users of the system.

Machine learning capability is planned for inclusion in the implementation of the tool by using decision tree algorithms to guide users in preference clarification. This is dependent on access to adequate data both in quality and quantity.

\subsection{Phase 4: End-user evaluation}

Potential users will be invited to evaluate the developed interactive tool in a 2-stage process.

\subsubsection{Stage 1: usability}

This stage will assess the usability of the interactive tool. Participants will be those involved in the focus groups and interviews in phase 2 of the study. They will provide feedback through a questionnaire and these will be analysed to inform the refinement of the interactive tool. In addition, adaptions of The System Usability Scale(SUS) (Brooke 1996) will be employed in this stage.

\subsubsection{Stage 2: SDM evaluation}

Following refinement, this stage will assess the effectiveness of the tool among new participants who will be recruited at this stage. Participants (clinicians, patients, relatives) will use the tool in actual medical deliberations and they will be invited to complete questionnaires to assess their perception of the tool's effectiveness in SDM, quality of decision, and usability. Validated instruments like the Decisional conflict scale (DCS) (O'Connor 1995) and SUS will be employed for this evaluation, including web analytic tools.

\subsection{Participants}

Four groups of participants have been identified for this study: patients, their relatives, oncologists, and clinical nurse specialists. They will be recruited 
from NHS sites in England and Pancreatic Cancer UK. NHS REC (Research Ethics Committee) approval process has begun and will be completed before data collection. A total of 120 participants will be required for the research study.

\section{INITIAL RESULTS}

The research study is currently in Phase 1. From an initial 3703 citations identified in 4 electronic databases (MEDLINE, PubMed, EMBASE, CENTRAL), 60 citations have been screened for eligibility based on the specified criteria.

The systematic review is registered on PROSPERO with record number: CRD42018087281.

\section{MAIN CONTRIBUTIONS BEING PROJECTED}

The main contributions projected from the study are listed below:

(i) Updated evidence on assessment of APC treatment through systematic review and NMA of reported RCTs.

(ii) Explorative study of the experiences and expectations of clinicians, patients and their relatives during the selection of appropriate treatments for APC and how these may influence the development and access of information resources for these groups of people.

(iii) Identification of features essential for the development of a web-based interactive tool to support SDM in APC treatment, which can potentially be transferred to other forms of cancer.

(iv) Assessment of the effectiveness of SDM using a web-based interactive tool for people with APC.

\section{ACKNOWLEDGEMENTS}

This research was funded by Bournemouth University and Poole Hospital NHS Foundation Trust. I would like to thank my supervisors, Dr Janet Scammell, Dr Nan Jiang, Professor Sam Porter, and Professor Tamas Hickish, for the helpful feedback on the manuscript for this paper.

\section{REFERENCES}

Balaban, E. P., P. B. Mangu, A. A. Khorana, M. A. Shah, S. Mukherjee, C. H. Crane, M. M. Javle, J. R. Eads, P. Allen, A. H. Ko, A. Engebretson, J. M. Herman, J. H. Strickler, A. B. Benson, 3rd, S. Urba and N. S. Yee (2016). Locally Advanced,
Unresectable Pancreatic Cancer: American Society of Clinical Oncology Clinical Practice Guideline. Journal Of Clinical Oncology: Official Journal Of The American Society Of Clinical Oncology 34(22): 2654-2668.

Beauchamp, T. L. and J. F. Childress (2013). Principles of biomedical ethics. New York, Oxford University Press.

Brooke, J. (1996). SUS-A quick and dirty usability scale. Usability evaluation in industry 189(194): 4-7.

Charles, C., A. Gafni and T. Whelan (1997). Shared decision-making in the medical encounter: what does it mean?(or it takes at least two to tango). Social science \& medicine 44(5): 681-692.

Charles, C., A. Gafni and T. Whelan (1999). Decision-making in the physician-patient encounter: revisiting the shared treatment decision-making model. Social science \& medicine 49(5): 651-661.

Coulter, A. and A. Collins (2011). Making shared decision-making a reality: no decision about me, without me, King's Fund.

Coulter, A., D. Stilwell, J. Kryworuchko, P. D. Mullen, C. J. Ng and T. van der Weijden (2013). A systematic development process for patient decision aids. BMC medical informatics and decision making 13(2): S2.

Creswell, J. W. and V. L. Plano Clark (2017). Designing and conducting mixed methods research, Los Angeles : SAGE, 2017.

Ducreux, M., A. S. Cuhna, C. Caramella, A. Hollebecque, P. Burtin, D. Goere, T. Seufferlein, K. Haustermans, J. L. Van Laethem, T. Conroy and D. Arnold (2015). Cancer of the pancreas: ESMO Clinical Practice Guidelines for diagnosis, treatment and follow-up. Ann Oncol 26 Suppl 5: v56-68.

Elwyn, G., A. Edwards, P. Kinnersley and R. Grol (2000). Shared decision making and the concept of equipoise: the competences of involving patients in healthcare choices. British Journal of General Practice 50(460): 892-+.

Elwyn, G., D. Frosch and S. Rollnick (2009). Dual equipoise shared decision making: definitions for decision and behaviour support interventions. Implementation Science 4(1): 75.

Elwyn, G., I. Kreuwel, M. A. Durand, S. Sivell, N. Joseph-Williams, R. Evans and A. Edwards (2011). How to develop web-based decision support interventions for patients: a process map. Patient education and counseling 82(2): 260-265. 
Elwyn, G., S. Laitner, A. Coulter, E. Walker, P. Watson and R. Thomson (2010). Implementing shared decision making in the NHS. British Medical Journal 341.

Ferlay, J., C. Partensky and F. Bray (2016). More deaths from pancreatic cancer than breast cancer in the EU by 2017. Acta Oncol 55(9-10): 1158-1160.

Freedman, B. (1987). Equipoise and the ethics of clinical research. New England Journal of Medicine,(th July).

Giacomin, J. (2014). What is human centred design? The Design Journal 17(4): 606-623.

Gresham, G. (2013). Informed Decision Making for Patients with Advanced Pancreatic Cancer Considering Chemotherapy: Development and Evaluation of a Clinical Decision Aid for Patients, Université d'Ottawa/University of Ottawa.

Hoffman, A. S., R. J. Volk, A. Saarimaki, C. Stirling, L. C. Li, M. Härter, G. R. Kamath and H. Llewellyn-Thomas (2013). Delivering patient decision aids on the Internet: definitions, theories, current evidence, and emerging research areas. BMC medical informatics and decision making 13(2): S13.

Hofmann, J. C., N. S. Wenger, R. B. Davis, J. Teno, A. F. Connors, N. Desbiens, J. Lynn and R. S. Phillips (1997). Patient preferences for communication with physicians about end-of-life decisions. Annals of internal medicine 127(1): 112.

Hui, D., M. De La Cruz, M. Mori, H. A. Parsons, J. H. Kwon, I. Torres-Vigil, S. H. Kim, R. Dev, R. Hutchins and C. Liem (2013). Concepts and definitions for "supportive care,"'best supportive care,"'palliative care," and "hospice care" in the published literature, dictionaries, and textbooks. Supportive Care in Cancer 21(3): 659-685.

Kamisawa, T., L. D. Wood, T. Itoi and K. Takaori (2016). Pancreatic cancer. Lancet 388(10039): 73-85.

Leighl, N. B., F. A. Shepherd, D. Zawisza, R. L. Burkes, R. Feld, J. Waldron, A. Sun, D. Payne, A. Bezjak and M. H. Tattersall (2008). Enhancing treatment decision-making: pilot study of a treatment decision aid in stage IV non-small cell lung cancer. Br J Cancer 98(11): 1769-1773.

Lu, G. and A. Ades (2004). Combination of direct and indirect evidence in mixed treatment comparisons. Statistics in medicine 23(20): 3105-3124.

Lumley, T. (2002). Network meta-analysis for indirect treatment comparisons. Statistics in medicine 21(16): 2313-2324.
Malvezzi, M., G. Carioli, P. Bertuccio, P. Boffetta, F. Levi, C. La Vecchia and E. Negri (2017). European cancer mortality predictions for the year 2017, with focus on lung cancer. Ann Oncol 28(5): 1117-1123.

Moher, D., A. Liberati, J. Tetzlaff, D. G. Altman and P. Group (2009). Preferred reporting items for systematic reviews and meta-analyses: the PRISMA statement. PLOS medicine 6(7): e1000097.

O'Connor, A. M. (1995). Validation of a Decisional Conflict Scale. Medical Decision Making 15(1): 25-30.

O'Connor, A. M., H. A. Llewellyn-Thomas and A. B. Flood (2004). Modifying unwarranted variations in health care: shared decision making using patient decision aids. Health Affairs: VAR63.

Office for National Statistics (2017) Cancer survival in England: adult, stage at diagnosis and childhood - patients followed up to 2016.

Ozanne, E. M., K. H. Schneider, D. Soeteman, N. Stout, D. Schrag, M. Fordis and R. S. Punglia (2015). onlineDeCISion. org: a web-based decision aid for DCIS treatment. Breast cancer research and treatment 154(1): 181-190.

Politi, M. C., M. D. Kuzemchak, K. A. Kaphingst, H. Perkins, J. Liu and M. M. Byrne (2016). Decision aids can support cancer clinical trials decisions: Results of a randomized trial. The oncologist 21(12): 1461-1470.

Stacey, D., C. L. Bennett, M. J. Barry, N. F. Col, K. B. Eden, M. Holmes-Rovner, H. LlewellynThomas, A. Lyddiatt, F. Légaré and R. Thomson (2011). Decision aids for people facing health treatment or screening decisions. Cochrane Database Syst Rev 10(10).

Stacey, D., F. Légaré, K. Lewis, M. J. Barry, C. L. Bennett, K. B. Eden, M. Holmes-Rovner, H. Llewellyn-Thomas, A. Lyddiatt and R. Thomson (2017). Decision aids for people facing health treatment or screening decisions. The Cochrane Library.

Stacey, D., R. Samant and C. Bennett (2008). Decision making in oncology: a review of patient decision aids to support patient participation. $C A$ : a cancer journal for clinicians 58(5): 293-304.

Standardization IOF (2010). Ergonomics of humansystem interaction: Part 210: Human-centred design for interactive systems, ISO.

Stiggelbout, A. M., T. Van der Weijden, M. P. De Wit, D. Frosch, F. Legare, V. M. Montori, L. Trevena and G. Elwyn (2012). Shared decision making: really putting patients at the centre of healthcare. BMJ 344: e256. 
Syrowatka, A., D. Krömker, A. N. Meguerditchian and R. Tamblyn (2016). Features of computerbased decision aids: systematic review, thematic synthesis, and meta-analyses. Journal of medical Internet research 18(1).

Taieb, J., A. L. Pointet, J. L. Van Laethem, B. Laquente, S. Pernot, F. Lordick and M. Reni (2017). What treatment in 2017 for inoperable pancreatic cancers? Annals of Oncology 28(7): 1473-1483.

Tempero, M. A., M. P. Malafa, M. Al-Hawary, H. Asbun, A. Bain, S. W. Behrman, A. B. Benson, 3rd, E. Binder, D. B. Cardin, C. Cha, E. G. Chiorean, V. Chung, B. Czito, M. Dillhoff, E. Dotan, C. R. Ferrone, J. Hardacre, W. G. Hawkins, J. Herman, A. H. Ko, S. Komanduri, A. Koong, N. LoConte, A. M. Lowy, C. Moravek, E. K. Nakakura, E. M. O'Reilly, J. Obando, S. Reddy, C. Scaife, S. Thayer, C. D. Weekes, R. A. Wolff, B. M. Wolpin, J. Burns and S. Darlow (2017). Pancreatic Adenocarcinoma, Version 2.2017, NCCN Clinical Practice Guidelines in Oncology. Journal of the National Comprehensive Cancer Network : JNCCN 15(8): 1028-1061.

Trevena, L. J., B. J. Zikmund-Fisher, A. Edwards, W. Gaissmaier, M. Galesic, P. K. Han, J. King, M. L. Lawson, S. K. Linder and I. Lipkus (2013). Presenting quantitative information about decision outcomes: a risk communication primer for patient decision aid developers. BMC medical informatics and decision making 13(2): S7.

Vincent, A., J. Herman, R. Schulick, R. H. Hruban and M. Goggins (2011). Pancreatic cancer. Lancet 378(9791): 607-620. 